\title{
$h$ HSS1: a novel secreted factor and suppressor of glioma growth located at chromosome 19q13.33
}

\author{
Katiana S. Junes-Gill • Timothy K. Gallaher • \\ Zoya Gluzman-Poltorak · Joseph D. Miller • \\ Christopher J. Wheeler · Xuemo Fan • Lena A. Basile
}

Received: 29 October 2009/ Accepted: 12 July 2010/Published online: 31 July 2010

(C) The Author(s) 2010. This article is published with open access at Springerlink.com

\begin{abstract}
The completion of the Human Genome Project resulted in discovery of many unknown novel genes. This feat paved the way for the future development of novel therapeutics for the treatment of human disease based on novel biological functions and pathways. Towards this aim, we undertook a bioinformatics analysis of in-house microarray data derived from purified hematopoietic stem cell populations. This effort led to the discovery of HSS1 (Hematopoietic Signal peptide-containing Secreted 1) and its splice variant HSM1 (Hematopoietic Signal peptidecontaining Membrane domain-containing 1). HSS1 gene is evolutionarily conserved across species, phyla and even kingdoms, including mammals, invertebrates and plants. Structural analysis showed no homology between HSS1 and known proteins or known protein domains, indicating that it was a truly novel protein. Interestingly, the human HSS1 ( $h$ HSS1) gene is located at chromosome 19q13.33, a genomic region implicated in various cancers, including
\end{abstract}

K. S. Junes-Gill · T. K. Gallaher · Z. Gluzman-Poltorak ·

L. A. Basile ( $\square)$

Neumedicines Inc, 133 N Altadena Dr. \#310,

Pasadena, CA 91107, USA

e-mail: basile@neumedicines.com

J. D. Miller

Department of Cell and Neurobiology, Keck School

of Medicine, University of Southern California, 1333 San Pablo

St. BMT401, Los Angeles, CA 90033-9112, USA

C. J. Wheeler

Department of Neurosurgery, Maxine Dunitz Neurosurgical Institute, Cedars-Sinai Medical Center, 8700 Beverly Blvd., Davis Rm. 2097, Los Angeles, CA 90048, USA

X. Fan

Department of Pathology and Laboratory Medicine,

Cedars-Sinai Medical Center, Los Angeles, CA 90048, USA malignant glioma. Stable expression of $h \mathrm{HSS} 1$ in gliomaderived A172 and U87 cell lines greatly reduced their proliferation rates compared to mock-transfected cells. $h$ HSS1 expression significantly affected the malignant phenotype of U87 cells both in vitro and in vivo. Further, preliminary immunohistochemical analysis revealed an increase in $h$ HSS1/HSM1 immunoreactivity in two out of four high-grade astrocytomas (glioblastoma multiforme, WHO IV) as compared to low expression in all four lowgrade diffuse astrocytomas (WHO grade II). High-expression of $h \mathrm{HSS} 1$ in high-grade gliomas was further supported by microarray data, which indicated that mesenchymal subclass gliomas exclusively up-regulated $h \mathrm{HSS} 1$. Our data reveal that HSS1 is a truly novel protein defining a new class of secreted factors, and that it may have an important role in cancer, particularly glioma.

Keywords HSS1 - Proliferation · Glioma - Chromosome 19q13.33 - Secreted protein $\cdot$ C19orf63

\section{Introduction}

The completion of the Human Genome Project marked a new era in our understanding of molecular genomics $[1,2]$. With this remarkable feat, previously unknown and uncharacterized proteins were revealed. The initial hope was that perhaps hidden within the vastness of the human genome were "truly novel proteins" with concomitant novel functions in biology. A "truly novel protein" is defined as one which has no known counterparts-that is, not just a newly-found member of a known protein family, but a novel protein that defines a new protein class or family [3]. The process of identifying and characterizing novel proteins defining a new protein class further opens up 
the possibility of discovering novel biological functions and pathways. This unveiling, in turn, could lead to significant progress in the treatment of human disease by defining new therapeutics and novel molecular targets.

In this paper, we provide the initial physical and functional characterization of a novel protein that defines a new protein class, designated as HSS1 (Hematopoietic Signal peptide-containing Secreted 1). HSS1 and its membranebound splice variant, HSM1 (Hematopoietic Signal peptide-containing Membrane domain-containing 1 ), form the first members of a novel protein class or family in that these proteins have no homology to any known proteins or any known protein domains. Given the novelty of HSS1, the elucidation of its function may provide insights into previously unknown biological functions.

The discovery of HSS1 started with a search for novel secreted proteins differentially expressed in hematopoietic stem cells (HSCs), implying a possible role in stem cell biology. We analyzed the gene expression profiles of three highly purified populations of murine HSCs [4]. This analysis led to the discovery of both mouse and human homologs of HSS1. But since HSS1 is a novel gene/protein that defines a novel protein class, no structural clues to its function were available. The only apparent functional clue was the gene locus of HSS1. Interestingly the gene related to human HSS1 ( $h \mathrm{HSS} 1$ ) maps to 19q13.33, a genomic site of putative but largely uncharacterized suppressor(s) of glioma and other cancers [5, 6]. This observation led us to investigate a possible role for $h \mathrm{HSS} 1$ in glioma malignancy. Thus, in this study we report the identification and characterization of a novel protein defining a new protein class, HSS1, as well as provide insight regarding its functional role in glioma tumor biology.

\section{Materials and methods}

\section{Isolation of HSS1 cDNA}

The murine form of HSS1 ( $m$ HSS1) was isolated via PCR from a lineage-negative mouse bone marrow cDNA library [7]. The human form of HSS1 (hHSS1) was isolated from a cDNA human testis library (Human MTC Multiple Tissue cDNA Panel II, Clontech). Primers were based upon predicted gene sequences and PCR products were sequenced to confirm the DNA sequence of both human and mouse HSS1 genes.

Cell culture and $h$ HSS 1 expression vector construct

Human embryonic kidney 293T and A172 glioma cell lines (ATCC, Manassas, VA, USA) were cultured in DMEM supplemented with 10\% FBS. The human U87 glioma cell line (ATCC HTB-14) was maintained in alpha-MEM supplemented with $10 \%$ FBS, non-essential amino acids, sodium pyruvate and sodium bicarbonate. The pTT3$h \mathrm{HSS} 1$ expression construct was kindly provided by Dr W. French Anderson. The $h \mathrm{HSS} 1 \mathrm{cDNA}$ was subcloned from pTT3 vector into the EcoRI and HindIII sites of pcDNA.3.1 mammalian expression vector (Invitrogen, Carlsbad, CA, USA). The construct had a 6-His tag in-frame fused at the C-terminal of $h \mathrm{HSS} 1$ gene. The resultant construct pCDNA3.1- $h$ HSS1 was verified by sequencing analysis.

\section{Transient transfection and immunoblotting}

Supernatants containing the $h \mathrm{HSS} 1$ protein were produced by transient transfection of 293T cells harvested after $48 \mathrm{~h}$ post-transfection using the Calcium Phosphate Transfection kit (Invitrogen) and $4 \mu \mathrm{g}$ of plasmid DNA purified with a Qiagen column (Qiagen, Valencia, CA, USA). Conditioned media from 293T cells were harvested, concentrated using a Macrosep centrifugal device (Pall, East Hills, NY, USA) and stored in aliquots at $4^{\circ} \mathrm{C}$. Protein expression was confirmed by Western blot in both supernatant and cell lysate prepared from $2 \times 10^{5}$ cells using a standard protocol and mass spectrometry. An anti-His (C-term)-HRP antibody (Invitrogen) allowed detection of $h \mathrm{HSS} 1$ protein by Western blot. Immunoreactive bands were visualized by colorimetric detection using TMB solution (Invitrogen). The glycosylation pattern of HSS1 was assessed by treating cell lysates with PNGase F, Sialidase and O-glycanase.

\section{Detection of $h$ HSS1-mRNA by reverse transcription (RT)-PCR}

Total RNA was isolated from A172 and U87 cell lines using the RNeasy minikit (Qiagen). The RT-PCR reaction was carried out by using SuperScrip III One-Step RT-PCR (Invitrogen) and $1 \mu \mathrm{g}$ of total RNA. The primers specific for HSS1 were: $5^{\prime}$-TCCTGCTCTTGCTGATGG-3' (forward); 5'-GAGACATAGCCACCAGCTTC-3' (Reverse). The conditions of the reaction were those suggested by the manufacturer. A GAPDH mRNA control was also amplified by PCR according to the manufacturer. PCR products were then checked via agarose gel electrophoresis.

\section{Selection of stable transfectants}

The glioblastoma-derived A172 and U87 cell lines were transfected as mentioned above, either with pcDNA3.1$h$ HSS 1 or with pcDNA3.1 empty vector. After $24 \mathrm{~h}$, $800 \mu \mathrm{g} \mathrm{ml}^{-1}$ of G-418 (Invitrogen) was added to the culture. Stable selection was performed for 4-8 weeks and the stably transfected clones were then expanded and cryopreserved in aliquots for further use. The expression of $h$ HSS1 mRNA was confirmed using RT-PCR. 
Quantitative proliferation assay

Cell viability was measured by cell counting using trypan blue exclusion on wild-type (untransfected cells), on pcDNA3.1 empty vector and pcDNA3.1- $h$ HSS 1 clones. U87 cells $\left(8 \times 10^{4}\right)$ were plated in triplicate in $10 \mathrm{~cm}$ plates and incubated at $37^{\circ} \mathrm{C}, 5 \% \mathrm{CO}_{2}$. After 6 days, the number of cells was quantified and the values were expressed as means. Also, $5 \times 10^{3}$ U87 cells were seeded in septuplicate in 96-well plates and after 3 days of incubation the number of cell aggregates formed was determined in the central field of each well. A172 cells $\left(8 \times 10^{4}\right)$ were plated in triplicate in $10 \mathrm{~cm}$ plates and after 7 days the cells were harvested and counted. To test the plating efficiency of A172 cells, $2 \times 10^{3}$ A172 cells were seeded in triplicate in $10-\mathrm{cm}$ plates. After 23 days, cell colonies were stained for $1 \mathrm{~h}$ with $0.001 \%$ neutral red (Sigma-Aldrich, St. Louis, MO, USA) in PBS, after that, plates were photographed. At least two experiments using each assay were performed.

Growth inhibition of cells

U87 and A172 wild-type cells, pcDNA3.1 and pcDNA$h$ HSS 1 cells were seeded at a density of $9 \times 10^{4} / 2 \mathrm{ml}$ medium in 6-well plates in triplicate. Over the course of 8 days, cells were harvested and counted by trypan blue dye exclusion.

\section{qRT-PCR}

For the analysis of $h \mathrm{HSS} 1$ expression in brain cancer tissue we used the TissueScan Brain Cancer Tissue qPCR Array I-HBRT102 (Origene Technologies, Rockville, MD), consisting of 48 human brain tissues normalized against $\beta$-actin gene. This array included meningiomas, oligodendroglioma and astrocytomas. Normal brain tissue was also evaluated using Human Brain Tissue qPCR Panel IHBRT101 (Origene Technologies), consisting of 24 human brain tissues normalized against GAPDH gene. qRT-PCR was performed using SYBR Green PCR master mix (Roche Diagnostics, Indianapolis, IN) using primers specific for $h$ HSS 1 (forward 5'-CAGCAGGATGGTACCTTGTC-3' and reverse $5^{\prime}$-GAGACATAGCCACCAGCTTC-3'). Relative mRNA levels of $h \mathrm{HSS} 1$ were calculated using the LightCycler 480 Relative Quantification Software 1.2 (Roche Diagnostics).

Immunocytochemical and immunohistochemical analysis

$h$ HSS1 was detected in U87 and A172 cells stably expressing $h \mathrm{HSS} 1$ and in formalin-fixed paraffin- embedded tissue sections of glioma tumors. U87 and A172 cells $\left(4 \times 10^{4}\right.$ cells $)$ were cultured overnight in 4 -well chamber slides coated with $5 \mu \mathrm{g} / \mathrm{ml}$ fibronectin. Cultures were fixed in cold methanol for $10 \mathrm{~min}$ at $-20^{\circ} \mathrm{C}$. Tissue sections from four low-grade diffuse astrocytomas (WHO grade II) and four high-grade astrocytomas (glioblastoma multiform, WHO grade IV) were incubated in AquaDepar (Biocare Medical, Concord, CA) reagent at $70^{\circ} \mathrm{C}$ for $10 \mathrm{~min}$. After deparaffinization, antigen retrieving was performed by using Reveal reagent (Biocare Medical). To block non-specific protein staining, cultures and sections were incubated in background sniper (Biocare Medical) for $10 \mathrm{~min}$. $h \mathrm{HSS} 1$ was detected by incubation for $2 \mathrm{~h}$ with a custom made rabbit polyclonal anti- $h$ HSS1 serum raised against synthetic peptide corresponding to amino acids 108-123 using 1:250 and 1:40 dilution for cultures and tissue sections, respectively (Genescript, Piscataway, NJ). This step was followed by incubation for $1 \mathrm{~h}$ at RT inImmPRESS reagent anti-rabbit Ig Peroxidase (Vector Laboratories, Burlingame, CA) and $30 \mathrm{~min}$ incubation in ImmPact AEC Peroxidase Substrate (Vector Laboratories). Slides were further counterstained with hematoxylin. Negative control included a stain containing all components except the $h \mathrm{HSS} 1$ primary antibody. The presence of $h$ HSS 1 was visualized by a reddish brown precipitate. The specificity of the $h$ HSS 1 polyclonal antibody was previously verified by Western blot analysis.

Anchorage-independent growth on soft agar

Medium containing U87 cells $\left(8 \times 10^{4}\right)$ was mixed with a $0.33 \%$ low-melting agarose top suspension, which was then layered onto a $0.5 \%$ bottom agarose. Each assay was performed in triplicate. The plates were incubated at $37^{\circ} \mathrm{C}, 5 \%$ $\mathrm{CO}_{2}$ for 23 days; after that, nine fields of each plate were photographed and colonies were counted manually.

Intracranial xenograft in immunodeficient mice

Tumorigenicity in vivo was determined by intracranial injection of U87 glioma-derived cells in male $\mathrm{Nu} / \mathrm{Nu}$ mice, age 6 weeks (Charles River Laboratories, Wilmington, MA, USA). Mice were quarantined for one week before initiation of the experimental procedure. Initially, mice were anesthetized with isoflurane $\left(\right.$ FORANE $\left.^{\circledR}\right)$. The skin was then cut using a scalpel and retracted for skull exposure. Skulls were punctured with a bone penetrator approximately $2 \mathrm{~mm}$ posterior to Bregma. After skull puncture, $10 \mu \mathrm{l}$ of a $1 \times 10^{6}$ cell suspension in PBS was injected with a $100 \mu \mathrm{l}$ syringe containing a blunt end needle. The skin was replaced over the skull puncture and given two stitches. Mice subsequently recovered from the anesthesia within minutes and became mobile and active. 
No apparent ill effects were observed immediately after the cell injection. Three groups of mice were studied: U87 wild-type cells $(\mathrm{N}=8), \quad$ pcDNA3.1 $(\mathrm{N}=5)$ and pcDNA3.1- $h \mathrm{HSS} 1(\mathrm{~N}=5)$. All mice were properly treated in accordance with guidelines of the Institutional Animal Care and Use Committee (IACUC) at the BATTS Laboratories facility (Northridge, CA, USA).

\section{Transcript expression profiling using microarray}

Affymetrix GeneChip Human Gene 1.0 ST Array was used to obtain transcript expression profiles in wild type (nontransfected), mock stable-transfected (pcDNA3.1 empty vector) and $h \mathrm{HSS} 1$-stable-transfected (pcDNA3.1- $h \mathrm{HSS} 1$ ) U87 cells. Exponentially growing U87 cells at growth curve day 4 were harvested by trypsinization and the total RNA was isolated using the RNeasy minikit (Qiagen). Further RNA characterization and chip analysis was carried out at the Functional Genomics Core of the City of Hope (Duarte, CA, USA). Samples were evaluated in triplicate. Expression values were determined using dChip (July 9, 2009 build). For glioma tissue microarray, 100 primary gliomas from MD Anderson Hospital patients (GEO accession \#GSE4271) were arranged into Mesenchymal, Proneural, and Proliferative subclasses according to Phillips et al. [8]. $h$ HSS1 probeset expression (Affymetrix HG-U133 probeset, 224727_at) was then assessed in each group from MAS5-normalized data, and statistical differences between groups evaluated using one-tailed student's $t$-test after adjustment for variance.

\section{Statistical analysis}

For the proliferation assays, two-tailed Student's $t$-tests were performed to establish the statistical significance of differences between control cells and $h \mathrm{HSS} 1$-expressing cells. Differences among groups in the growth curve analysis were determined by two-way analysis of variance (ANOVA) with Tukey's test for pairwise post-hoc comparisons. Survival analysis was performed by the KaplanMeier test. Differences were considered statistically significant when $P<0.05$.

\section{Results}

Bioinformatics, cloning and sequencing of HSS1 and HSM1

Using Affymetrix microarray analysis, approximately 900 highly and/or differentially expressed genes were identified from three subsets of murine HSC populations [4]. Probe sets with significant expression in the HSC subsets of unknown gene products were subjected to BLAT analysis and examined for correspondence to novel in silico predicted genes. Predicted genes identified in this manner were then subjected to analysis for signal peptides using SignalP (http://www.cbs.dtu.dk/services/SignalP/) and the absence of a transmembrane domain, using TMHMM (http://www.cbs.dtu.dk/services/TMHMM/). In this manner, novel secretable proteins were identified. Gene expression corresponding to one probe (96320_at) found in all three stem cell subsets identified a downstream untranslated region of a predicted gene on mouse chromosome 7 (GenScan chr7_6.156) and on human chromosome 19 (GenScan NT_011109.821). The respective mouse and human gene predictions specified proteins with $86 \%$ identity to each other, neither of which was existent in the databank. These mouse and human genes predicted a signal peptide sequence, but no transmembrane domain, indicating that the sequences coded for secreted proteins. Both mouse and human genes were cloned from their respective mouse and human cDNA libraries via PCR using primers based upon the gene predictions. The clones were then sequenced and the predicted sequences were confirmed for both human and mouse. The secretable form of the gene was designated HSS1 (Fig. 1a). Additionally, GenBank entries indicated the presence of a splice variant of HSS1 containing a predicted membrane spanning domain. This isoform was also amplified by PCR from the cDNA libraries, confirmed for both human and mouse and was designated HSM1, the membrane associated splice variant of the gene (Fig. 1a). The mouse and human gene ID's are 69683 and 284361, respectively.

The full-length cDNA sequence of HSS1 consists of approximately $1.9 \mathrm{~kb}$ containing an open reading frame of 789 bp. This sequence was submitted to the NCBI database and assigned a GenBank accession number, namely AY761095. The genomic structure of HSS1, as well as HSM1, is composed of seven exons and six introns covering a minimum of $6.8 \mathrm{~kb}$ (Fig. 1b). The last exon is alternatively spliced resulting in either HSS1 or HSM1. The two forms of the gene are encoded at chromosome 19q13.33. Mouse and human HSS1/HSM1 share homology with ortholog genes in numerous species (Fig. 2a). The Beijing Genomics Institute Tree Families Database, for example, includes 45 different species to date as part of the family (TF314052). Thus, the gene is evolutionarily conserved across species, phyla and kingdoms, including mammals, invertebrates and plants.

HSS1 and HSM1 do not have sequence homology to known naturally occurring proteins

Since HSS1 was identified solely by bioinformatic analysis, and HSM1 was only one gene out of thousands of 
Fig. 1 a Protein sequence alignment for HSS1 and HSM1. Homology between the two splice variants in mouse and human are shown. Identical residues are indicated by asterisks. Shaded regions represent as follows: predicted signal peptide (light grey); predicted $\mathrm{N}$-and $O$-glycosylation sites at the amino acid positions 182 and 198 (black box); predicted transmembrane domain for hHSM1 (dark grey, note it contains the ultimate intronexon splice boundary which gives rise to the different forms of the gene): intron-exon junctions (bold underlined). b Intron-exon arrangement of $h$ HSS1/HSM1. Each HSS1 and HSM1 contains seven exons. The last exon differs in both forms of the protein. Small arrows indicate PCR primer sites. TGA: stop codon. The two forms of the gene are encoded at locus $19 \mathrm{q} 13.33$

\section{a}

hHSM1 mHSM1 hHSS1 mHSS1

hHSM1 mHSM1 hHSS1 mHSS1

hHSM1 mHSM1 hHSS1 mHSS1

hHSM1 mHSM1 hHSS 1 mHSS1

hHSM1 mHSM1 hHSS1 mHSS1

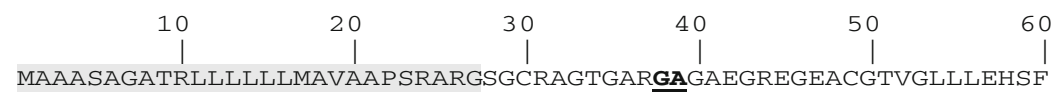
MVAAGAGVTRLLVLLLMVAAAPSRARGSGCRVGASARGTGADGREAEGCGTVALLLEHSF MAAASAGATRLLLLLLMAVAAPSRARGSGCRAGTGARGAGAEGREGEACGTVGLLLEHSF MVAAGAGVTRLLVLLLMVAAAPSRARGSGCRVGASARGTGADGREAEGCGTVALLLEHSF

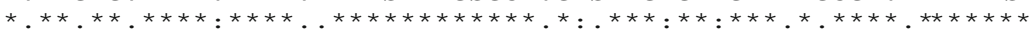

$\begin{array}{rrrrrr}70 & 80 & 90 & 100 & 110 & 120 \\ \mid & \mid & \mid & \mid & \mid\end{array}$
EIDDSANFRKRGSLLWNQQDGTLSLSQRQLSEEERGRLRDVAALNGLYRVRIPRRPGALD ELGDGANFOKRGLLLWNOODGTLSATOROLSEEERGRLRDVAAVNGLYRVRVPRRPGTLD EIDDSANFRKRGSLLWNQQDGTLSLSQRQLSEEERGRLRDVAALNGLYRVRIPRRPGALD ELGDGANFQKRGLLLWNQQDGTLSATQRQLSEEERGRLRDVAAVNGLYRVRVPRRPGTLD

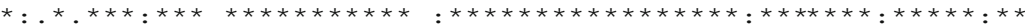

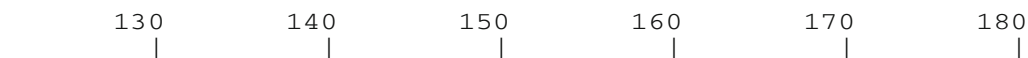<smiles>C1CCCC1</smiles><smiles></smiles><smiles>C1CCC1</smiles>

GLEAGGYVSSFVPACSLVESHLSDQLTLHVDVAGNVVGVSVVTHPGGCRGHEVEDVDLEL GSEAGGHVSSFVPACSLVESHLSDQLTLHVDVAGNVVGLSVVVYPGGCRGSEVEDEDLEL GLEAGGYVSSFVPACSLVESHLSDQLTLHVDVAGNVVGVSVVTHPGGCRGHEVEDVDLEL GSEAGGHVSSFVPACSLVESHLSDQLTLHVDVAGNVVGLSVVVYPGGCRGSEVEDEDLEL

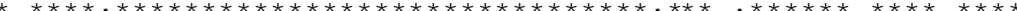

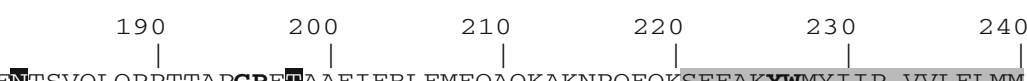
NTS SVLQPPTTAPGPETAAFIERLEMEQAQKAKNPQEQKSFFAKYWMYIIP-VVLFLMM FNTSVQLRPPSTAPGPETAAFIERLEMEQAQKAKNPQEQKSFFAKYWMYIIP-VVLFLMM FNTSVQLQPPTTAPGPETAAFIERLEMEQAQKAKNPQEQKSFFAKYWHI ILGGAVLLTAL FNTSVQLRPPSTAPGPETAAFIERLEMEQAQKAKNPQEQKSFFAKYWHLILGGAVLLTAI $* * * * * * *: * *: * * * * * * * * * * * * * * * * * * * * * * * * * * * * * * * * * * * *):{ }^{* *}::$

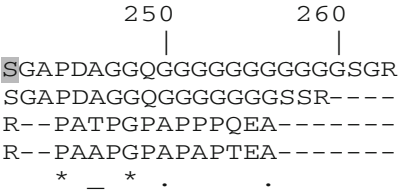$$
\text { * }-{ }^{*} \text {. }
$$

b

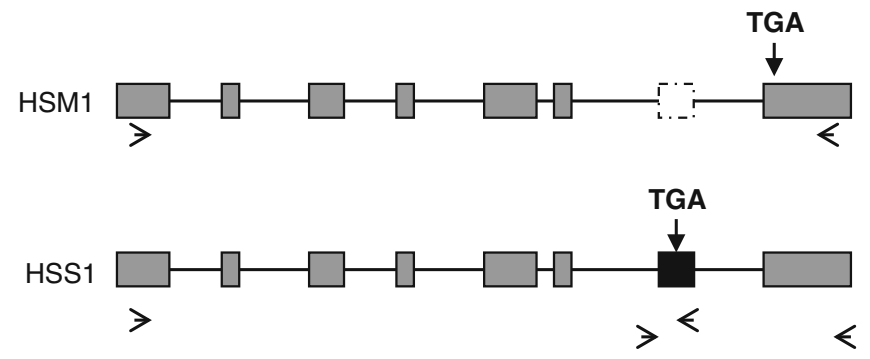

uncharacterized genes which had been sequenced in various EST or transcriptomic projects, no function could be attributed a priori to these genes. Towards the aim of elucidating a function for the proteins encoded by the HSS1 gene, we searched extensively for homology between HSS1/HSM1 and proteins with known functions. We also performed searches to ascertain whether HSS1/ HSM1 contained domains common to a known protein and/ or protein domain family. Using publicly available algorithms to identify protein domains and overall structure, we failed to find any homology with HSS1 or HSM1.

We then used a proprietary algorithm (Eidogen-Sertanty Inc., Oceanside, CA, USA) based on a 3-D, crystallographic protein database to align protein sequences of unknown structure. Interestingly, this search revealed homology to only one protein in the database. This protein was a non-natural, computationally designed protein, designated as TOP7 [9]. From this analysis, we determined that a region common to HSS1 and HSM1 share structural homology with TOP7 at the 95\% confidence level (Fig. 2b and $\mathrm{c}$ ).

HSS1 is a novel secreted protein with a complex glycosylation pattern

Based on the primary sequence of HSS1, SignalP analysis showed that HSS1 has a predicted signal peptide, but according to TMHMM no transmembrane domain. To further confirm its potential secretory properties, $h \mathrm{HSS} 1$ was expressed in $293 \mathrm{~T}$ cells as a construct containing a $6 \times$ His tag at its carboxyl terminal. Western blot analysis using an anti- $6 \times$ His antibody confirmed that HSS1 is a secreted protein, as it was detected in the supernatant of transiently transfected $293 \mathrm{~T}$ cells (Fig. 3a and b). Wild 
Fig. 2 a Phylogram of HSS1/ HSM1 protein sequences. Alignment of HSS1/HSM1 protein sequences from 24 species using ClustalW2 ( http://www.ebi.ac.uk/Tools/ clustalw2/index.html). HSS1/ HSM1 has identified orthologs in at least 45 species, of which 24 are shown. b and c Overlay of TOP7 fold with the HSS1/ HSM1 predicted fold. b Alignment of TOP7 (blue) and HSS1/ HSM1 (green) $\beta$-sheets (amino acids 54-159); $\mathbf{c}$ alignment of TOP7 (red) and HSS1/HSM1 (green) alpha-helices. The predicted probability of these two domains aligning by chance is $P<0.05$

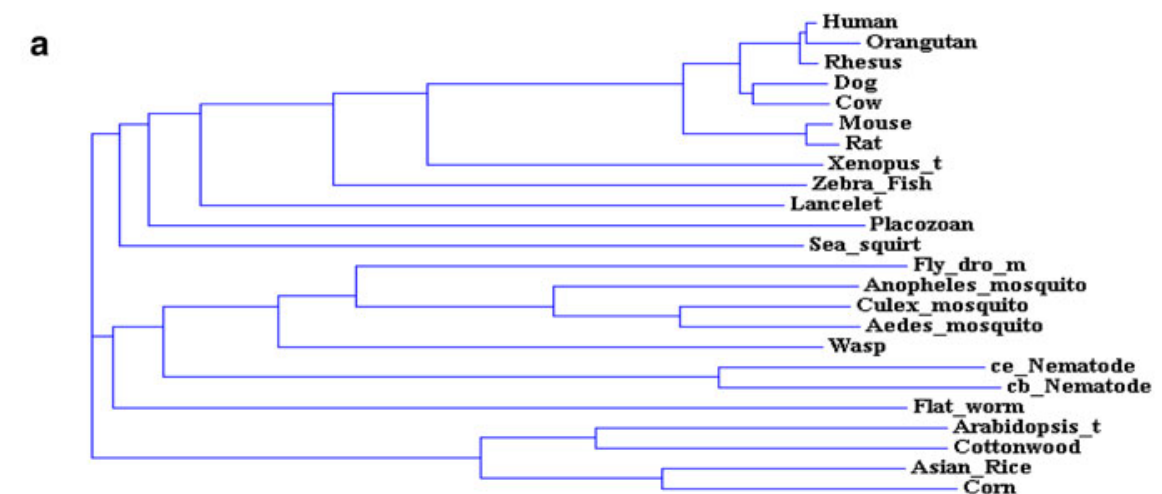

b

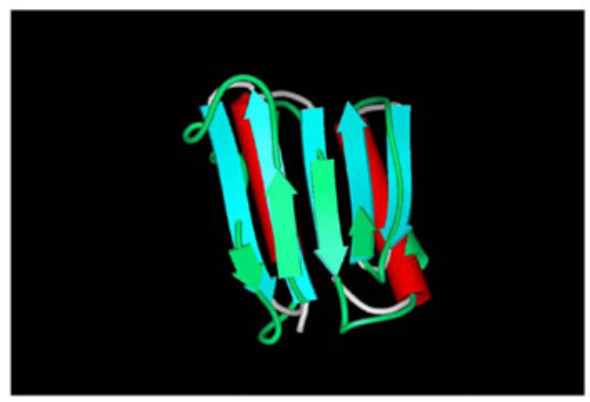

C

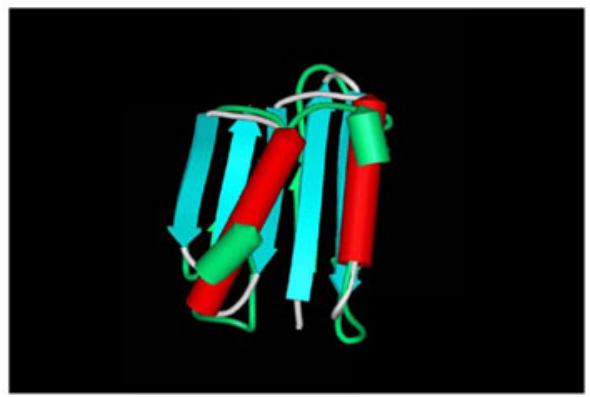

type 293T cells (Fig. 3b) and the cells transfected with empty vector (data not shown) yielded no protein via Western blot analysis.

We next wanted to confirm if HSS1 is glycosylated, as the predicted sequence presented two possible glycosylation sites at residues 182 and 198 (Fig. 1a) based on NetNGlyc and NetOGlyc analysis (http://www.cbs.dtu.dk/ services/). Thus, cleavage of HSS1 by glycolytic enzymes was performed on the cell lysate and supernatants containing HSS1. The apparent molecular weight of unglycosylated HSS1 from analysis of the His tag ladder (Fig. 3a, lane 1) is approximately $30 \mathrm{kDa}$ (Fig. 3a, lanes 2-5). The theoretical molecular weight of the mature (i.e. signal peptide cleaved) HSS1 is $24,171 \mathrm{Da}$. The majority of the protein in the cell lysate did not appear to be glycosylated as enzyme cleavage did not significantly change the apparent molecular weight. However, for HSS1 that is secreted and found in the supernatant, cleavage by various glycolytic enzymes incrementally decreased the apparent molecular weight of the protein (Fig. 3a, lanes 6-13), thus suggesting a complex glycosylation pattern.

$h$ HSS1 has a growth inhibitory effect in glioma cells

Following our initial characterization of the physical properties of HSS1, we next sought to determine a function for this novel protein. Since Mitelman Breakpoint Data revealed that $h \mathrm{HSS} 1$ is located in a chromosomal region known to have aberrations or deletions in a variety of cancers, including various gliomas [10], we first investigated whether $h \mathrm{HSS} 1$ would affect the malignant proprieties of two well-studied human glioma-derived cell lines, namely A172 and U87. These glioblastoma cell lines were chosen for study because previously they were found to have deletions in a region corresponding to the gene locus of $h \mathrm{HSS} 1$ at 19q13.33 [11]. However, because $h \mathrm{HSS} 1$ is expressed in normal brain tissues [12], we checked whether these cell lines were expressing $h \mathrm{HSS} 1$ by using gene specific primers in a one-step RT-PCR reaction. We found that neither $h$ HSS 1 nor $h$ HSM 1 mRNA was detectable in these glioblastoma cell lines (Fig. 4a, lanes 1 and 4).

We then stably transfected A172 and U87 with a construct carrying $h \mathrm{HSS} 1$ cDNA (pcDNA3.1- $h \mathrm{HSS} 1$ ) or with the empty vector as control. The expression of $h \mathrm{HSS} 1$ following transfection was confirmed by using RT-PCR. The results showed that while $h \mathrm{HSS} 1$ mRNA was not detectable in non-transfected wild-type and mock-transfected cells, the selected pcDNA3.1- $h$ HSS1-transfected clones stably expressed the $h \mathrm{HSS} 1 \mathrm{mRNA}$ (Fig. 4a, lanes 2 and 5).

During the selection of the stable clones, we observed that the growth of the pcDNA3.1-hHSS1 transfected cells was dramatically decreased relative to mock-transfected clones. Thus, cell counting and plating efficiency were performed to evaluate the relative proliferation of clones transfected with pcDNA3.1- $h$ HSS1 or empty vector. The results obtained showed that A172- and U87-hHSS1expressing glioblastoma cells decreased in cell number by 


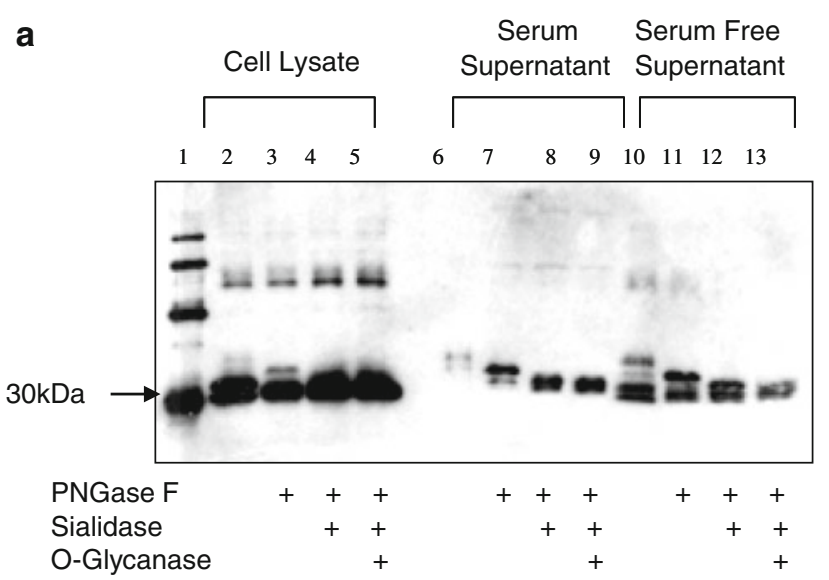

b

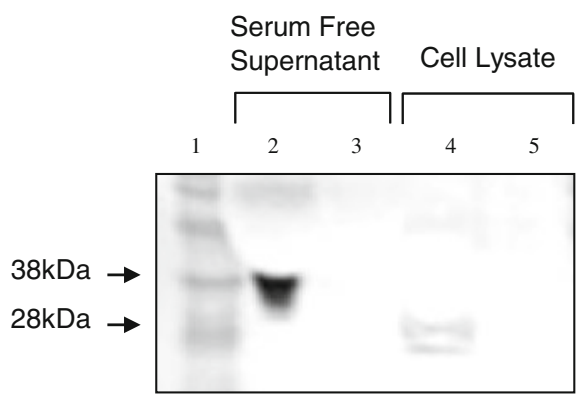

Fig. 3 HSS1 is secreted and multi-glycosylated a Western blot analysis obtained from $293 \mathrm{~T}$ cells transfected with the pTT3- $h$ HSS 1 construct in serum or serum-free medium. The protein was detected with anti-His-tag antibody. Samples were also digested with enzymes that selectively cleave glycosylated proteins. His Tag ladder (lane 1); denatured cell lysate (lane 2); cell lysate treated with different enzymes (lanes 3, 4 and 5); denatured supernatant from cells grown in medium with serum (lane 6 ); supernatant with serum treated with different enzymes (lanes 7, 8 and 9); denatured supernatant from cells grown in serum-free medium (lane 10); supernatant serum-free treated with different enzymes (lanes 11, 12 and 13). b Western blot analysis from $293 \mathrm{~T}$ cell. Serum free supernatant and cell lysate of $293 \mathrm{~T}$ cells transiently transfected with $h \mathrm{HSS} 1$ (lanes 2 and 4 , respectively). Serum free supernatant and cell lysate of wild type 293 T cells (lanes 3 and 5, respectively)

3-fold compared to mock-transfected cells (two-tailed independent Student's $t$-test, $P<0.001$ ) (Fig. $4 \mathrm{~b}$ and d).

In addition to reduction in proliferation, expression of the $h \mathrm{HSS} 1$ gene in U87 cells induced morphological changes. We observed that cell aggregate formation in U87-mocktransfected cells were discernible (Fig. 4f), probably reflecting loss of contact inhibition in these cells. Cell aggregates can be viewed as clumps of cells that accumulate over an initial monolayer. This phenotype in U87 cells was also reported in a previous study of the Smac gene and its regulation of tumor cell viability [13]. We next evaluated the number of cell aggregates formed between $h \mathrm{HSS} 1$ expressing cells and mock-transfected cells. The number of cell aggregates formed in U87 cells expressing $h$ HSS 1 was 5.9 times less relative to mock-transfected cells (two-tailed independent Student's t-test, $P<0.001$ ) (Fig. 4e). The number of cells counted in this experiment was $1.32 \times 10^{5}$ $\left(\right.$ pcDNA3.1) and $3.35 \times 10^{4}$ (pcDNA3.1-hHSS1) (twotailed independent Student's $t$-test, $P<0.02$ ).

Since A172 cells do not display cell aggregate formation, we chose to assess the difference in plating efficiency for this cell line as an additional measure of proliferation and ability to grow at low density. We observed that the number of colonies formed by A172 cells expressing $h \mathrm{HSS} 1$ was dramatically decreased compared to the mocktransfected (Fig. 4c), indicating that hHSS1 expression efficiently reduced clonogenic cell survival.

Since we have verified the expression of $h \mathrm{HSS} 1$ in these cell lines only by mRNA levels, we then checked the expression of $h \mathrm{HSS} 1$ at the protein level by immunochemical analysis. Figure 5 shows immunocytochemical analysis of U87 (Fig. 5a) and A172 (Fig. 5b) cell lines. No obvious staining was detectable in non-transfected and empty-vector transfected U87 cells (Fig. 5a.1-2). Immunoreactivity was evident throughout the cells overexpressing $h \mathrm{HSS} 1$, confirming that its transcript in U87 and A172 (Fig. 5a.3 and 5b.6, respectively) stable cells was translated into immmocytochemically detectable $h \mathrm{HSS} 1$ protein. Conversely, endogenous $h \mathrm{HSS} 1$ expression in some cells was detected in both non-transfected and emptyvector transfected A172 cells (Fig. 5b.4-5). However, the A172 $h$ HSS1-transfected cells expressed higher levels of $h$ HSS1 protein compared to the controls (Fig. 5b.6). Nuclear staining was observed in A172 cells, suggesting that $h \mathrm{HSS} 1$ may also localize inside the nucleus of these cells. Thus, $h$ HSS1 could have both intra and extracellular properties as has been reported for FGFs and epidermal growth factor (EGF) proteins [14]. Further experiments are needed to confirm this finding.

To further examine the growth properties of the $h \mathrm{HSS} 1-$ expressing cells, we constructed a growth curve for each cell line in order to determine whether $h \mathrm{HSS} 1$ overexpression increased the cell doubling time. The growth rate was significantly affected by $h \mathrm{HSS} 1$ overexpression in both A172 cells [main effect of cell type, $F(2,135)=98.61$, $P<0.001$ ] and U87 cells [main effect of cell type, $F(2$, $135)=51.36, P<0.001]$. A significant growth inhibition effect was observed after day 3 of experiment for both cell lines: A172 cells [cell type $\mathrm{X}$ day interaction, $F(16$, $135)=6.84, P<0.001]$, U87 cells [cell type $\mathrm{X}$ day interaction, $F(16,135)=3.45, P<0.001]$. Moreover, the A172 wild-type and mock-transfected cells showed a doubling time of 27 and $30.5 \mathrm{~h}$, respectively, whereas the A172 $h$ HSS1-expressing cells showed a doubling time of 40 h (Fig. 6a). Similarly, while U87 wild-type and mocktransfected cells had a doubling time of 27.4 and $27.6 \mathrm{~h}$, respectively, the U87 $h \mathrm{HSS} 1$-expressing cells had a doubling time of $32.1 \mathrm{~h}$ (Fig. 6b). Taken together, these results 
a

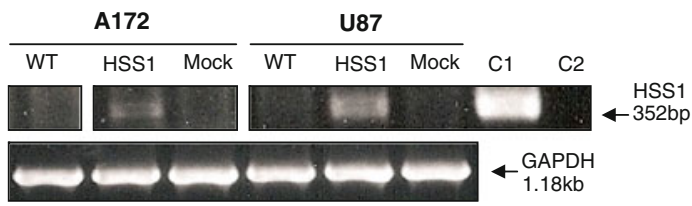

b

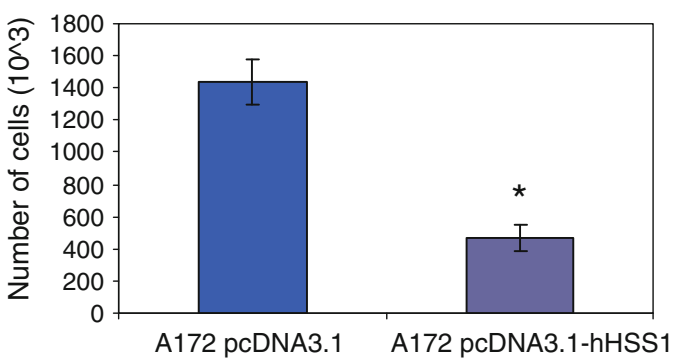

C

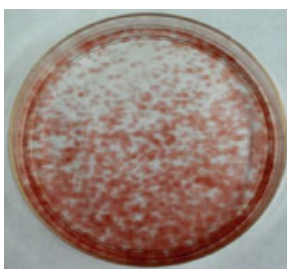

A172 pcDNA3.1

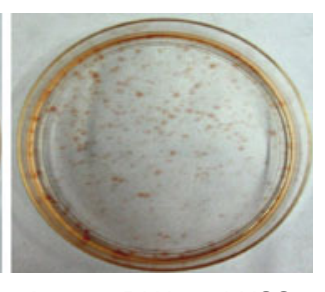

A172 pcDNA3.1-hHSS1
Fig. 4 Growth inhibitory effect of $h \mathrm{HSS} 1$ in glioma cells a RT-PCR analysis of the clones stably transfected with pcDNA3.1- $h$ HSS 1 or pcDNA3.1 empty vector selected for the experiments. A172 and U87 cell lines do not express detectable levels of $h \mathrm{HSS} 1$ (or $h \mathrm{HSM} 1$ ) (lanes 1 and 4, upper panel). PCR product from the stable clones expressing HSS1 (lanes 2 and 5) and mock-transfected cells (lanes 3 and 6); $C 1$, positive control: $100 \mathrm{ng}$ of pcDNA3.1- $h \mathrm{HSS} 1$ plasmid; $C 2$, negative control: reaction reagent only. b A172 pcDNA3.1 and pcDNA3.1- $h$ HSS 1 transfected cells were seeded $\left(8 \times 10^{4}\right.$ cells $)$ and harvested after 7 days for cell counting by trypan blue exclusion. c $h$ HSS 1 expression suppresses colony formation in A172. A172 pcDNA3.1 and pcDNA3.1-hHSS1 transfected cells were seeded

indicate that $h \mathrm{HSS} 1$, a novel secreted protein in a new protein class, has a growth-inhibitory effect in malignant glioma cells.

$h$ HSS 1 suppresses the malignant phenotype of U87 cells in vitro and in vivo

Changes in cell morphology are associated with malignant transformation in many epithelial tumor cells. Furthermore, malignantly transformed cells have the ability to grow in the absence of cell anchorage. To evaluate whether the morphological changes induced by $h \mathrm{HSS} 1$ expression (Fig. 4f) correlate with reduced malignancy in these cells, we compared the ability of U87 cells carrying either the empty vector or expressing $h \mathrm{HSS} 1$ to grow in an anchorage- d

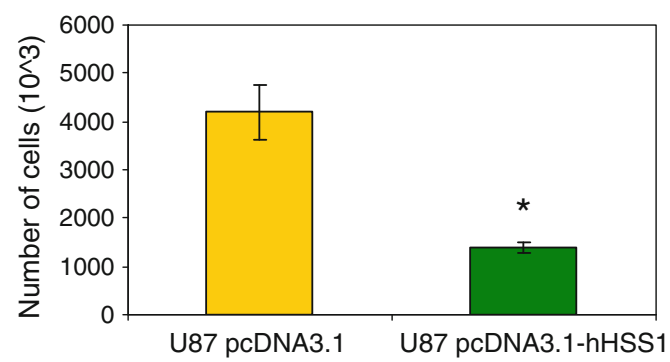

e

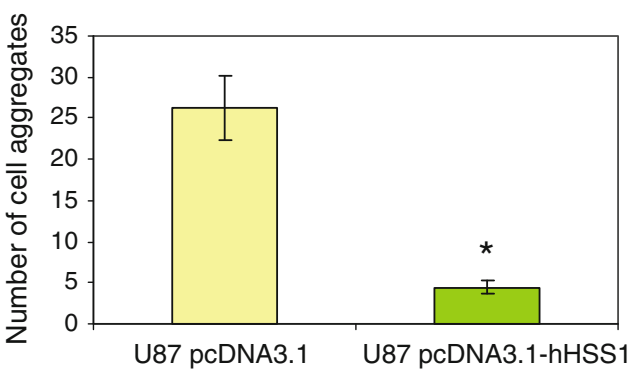

f

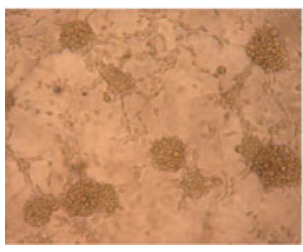

U87 pcDNA3.1

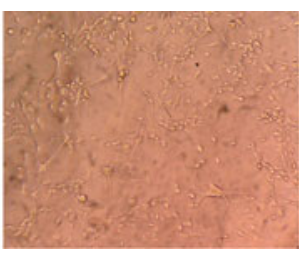

U87 pcDNA3.1-hHSS1 $\left(2 \times 10^{3}\right.$ cells), and after 23 days cells were stained with neutral red. d U87 pcDNA3.1 and pcDNA3.1- $h$ HSS1 transfected cells were seeded $\left(8 \times 10^{4}\right.$ cells $)$ and harvested after 6 days for cell counting by trypan blue exclusion. e and $\mathbf{f} h$ HSS 1 expression decreases U87 cell aggregation in culture. e U87 cells $\left(5 \times 10^{3}\right)$ were seeded in septuplicate in 96-well plates and incubated for 3 days, and then cell aggregates were photographed and counted. f $h \mathrm{HSS} 1$-expressing cells presented a much flatter shape while the control cells typically grew in cell aggregates. All results are presented as grand mean \pm SEM of two independent experiments. $* P<0.05$, two-tailed independent Student's $t$-test

independent manner. Results showed that empty vector control cells formed numerous colonies in soft agar compared to $h \mathrm{HSS} 1$-expressing cells (Fig. 7a). Moreover, the sizes of the colonies of empty vector control cells were markedly larger than those of cells expressing $h \mathrm{HSS} 1$ (Fig. 7b). These results suggest that stable expression of $h \mathrm{HSS} 1$ in U87 cells in which $h \mathrm{HSS} 1$ expression previously had been undetected produces a potentially less malignant phenotype by restoring the normal cell property of contact inhibition.

We also evaluated the ability of transplanted U87 cells to affect survival in immunocompromised mice (A172 cells were not studied as these cells are not tumorigenic in mice). Mice implanted with U87 $h$ HSS1-expressing cells survived significantly longer, as compared to those implanted with 
Fig. 5 Immunocytochemical localization of $h \mathrm{HSS} 1$ in cultured glioma-derived cells. a: 1, U87 wild type cells; 2 , pcDNA3.1-transfected U87 cells; 3 , pcDNA3.1- $h$ HSS1 transfected U87 cells. Pictures shown represent cells stained using anti-rabbit antibody against $h \mathrm{HSS} 1$. b: 4, A172 wild type cells; 5 , pcDNA3.1transfected A172 cells; 6, pcDNA3.1- $h$ HSS1 A172 transfected cells. Red arrows nuclear staining. Images are shown in the same magnification $(200 \times)$
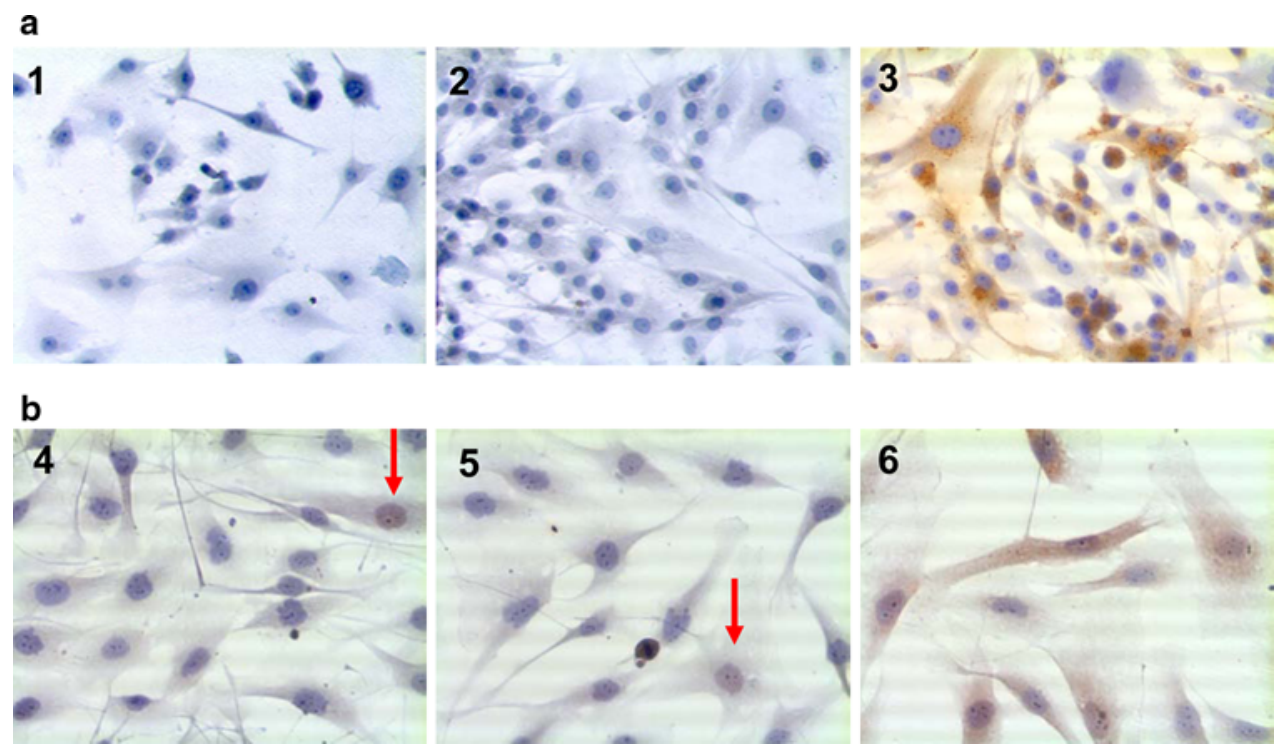

Doubling time A172: $27 \mathrm{~h}$

pcDNA3.1: $30.5 \mathrm{~h}$ pCDNA3.1-hHSS1: 40h
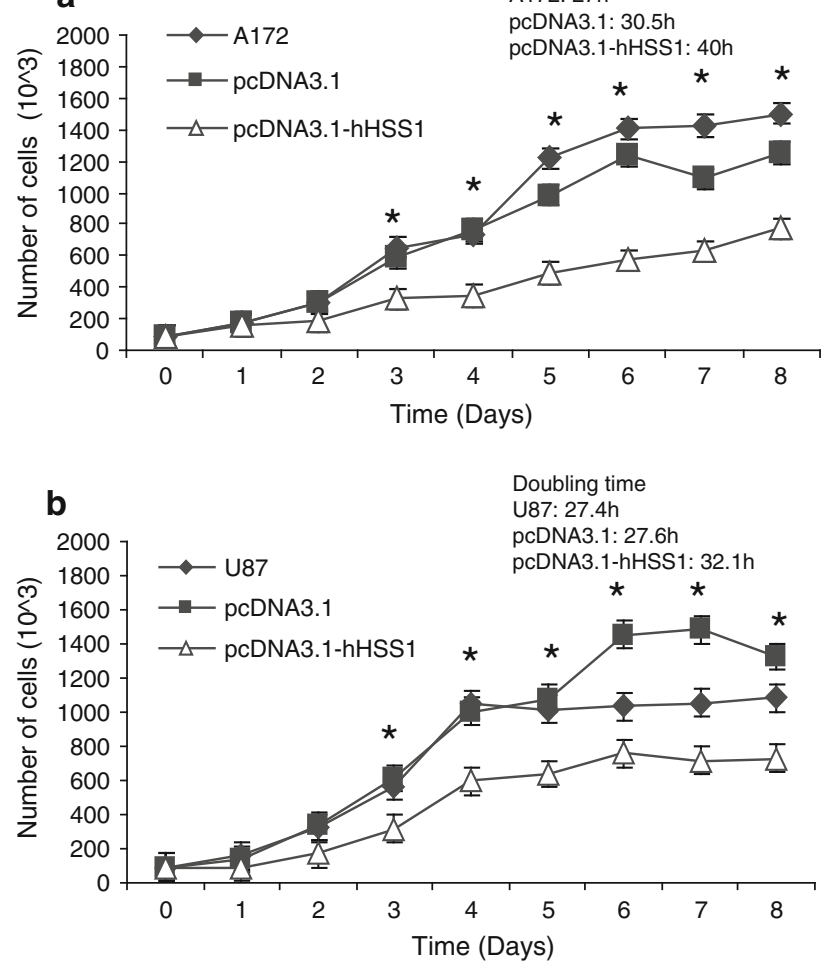

Fig. $6 h$ HSS1 expression inhibits A172 and U87 cell doubling. a and b A172 and U87 cells (wild-type, pcDNA3.1, pcDNA3.1- $h$ HSS1) were cultured at nine different time points, harvested and counted by trypan blue exclusion. a $h \mathrm{HSS} 1$ significantly inhibited the proliferation of A172 cells after 3 days in culture $(* P<0.05$, post-ANOVA pairwise Tukey test). b $h$ HSS 1 significantly inhibited the proliferation of U87 cells after 3 days in culture $(* P<0.05$, post-ANOVA pairwise Tukey test). Results are shown as grand mean \pm SEM of two independent experiments

U87 wild-type cells or cell carrying the empty vector (78 days vs 34 and 30 days for mock-transfected and wildtype cells, respectively; $P<0.0001 ; \log$-rank). This result suggests that $h \mathrm{HSS} 1$ decreases the malignancy of U87 cells in vivo (Fig. 7c).

$h \mathrm{HSS} 1$ alters gene expression profile in stably-transfected U87 cells

The observed growth inhibitory effect of $h \mathrm{HSS} 1$, as well as its suppression of tumorigenicity in vitro and in vivo, raises the question of the molecular basis for such phenotypical changes. Comparative gene expression profiling using Affymetrix GeneChip Human Gene 1.0 ST of wild-type (non-transfected), mock-stable-transfected (pcDNA3.1 empty vector) and $h \mathrm{HSS} 1$-stable-transfected (pcDNA3.1$h$ HSS1) U87 cells allowed us to address this question.

Expression profiles of U87, mock and $h \mathrm{HSS} 1$-transfected stable cell lines were obtained and compared by two-tailed paired Student's $t$-test. Differential expression due to the presence of $h \mathrm{HSS} 1$ was defined as being statistically significant $(P<0.05)$ for the $h$ HSS1-transfected cells compared to both U87 wild-type and the mock transfected controls with the added criterion that difference in gene expression between U87 wild-type and mock transfected cells must not be statistically significant $(P>0.05)$. After these stringent criteria were met, 491 genes were found to have altered expression levels of at least two-fold. A volcano plot is shown in Fig. 8. Among these genes were $h$ HSS 1 itself which exhibited more than 10-fold increase in expression in the $h \mathrm{HSS} 1$ stable-transfected cells compared to mock stable-transfected $(P=0.00013)$ and U87 wildtype cells $(P=0.00015)$. The expression levels of $h \mathrm{HSS} 1$ between U87 wild-type and the mock stable-transfected cells was not statistically significant $(P=0.39)$. These findings for $h \mathrm{HSS} 1$ in the expression profile analysis are indicative of the integrity of the analysis. Overall, 166 probeset defined genes were up-regulated and 325 were down- 


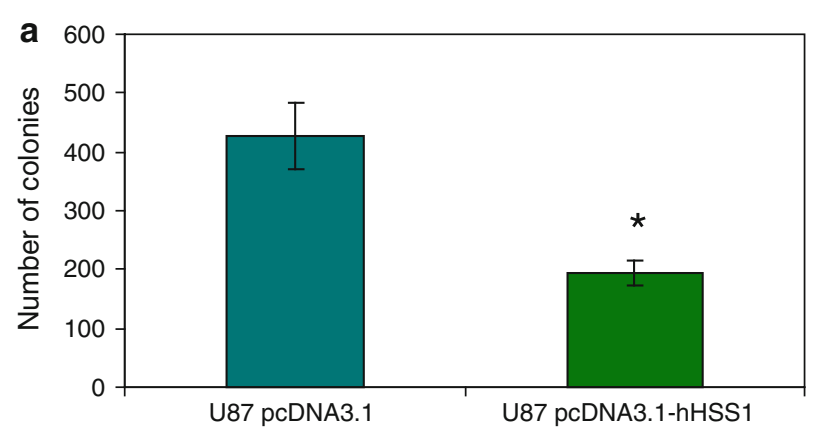

b

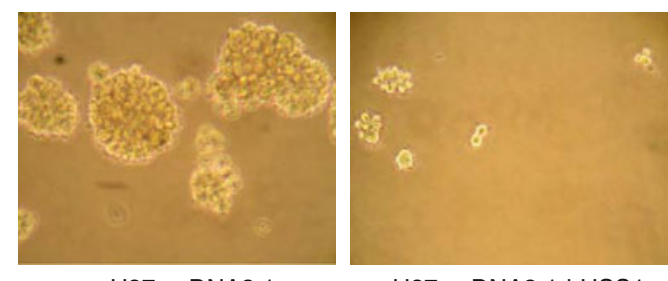

U87 pcDNA3.1

U87 pcDNA3.1-hHSS1

C

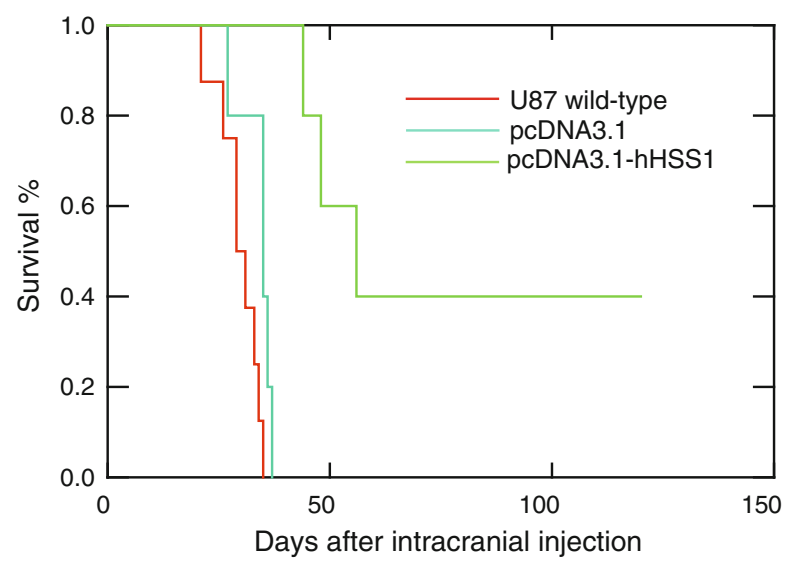

Fig. $7 h$ HSS1 suppresses anchorage-independent growth and tumorigenicity of U87 cells. a U87 cells (pcDNA3.1, pcDNA3.1- $h$ HSS1) were seeded on top of soft agar in $10 \mathrm{~cm}$ plates and the number of colonies formed in soft agar were counted after 23 days of incubation. Results are expressed as grand mean \pm SEM and are representative of two independent experiments. $* P<0.05$, Student's $t$-test, pcDNA3.1- $h$ HSS1 versus U87 wild-type or versus mock-transfected cells. b Micrograph of U87 colonies grown in soft agar: pcDNA3.1: mock-transfected cells, pcDNA3.1- $h \mathrm{HSS} 1$ : cells stably expressing $h$ HSS1. c Kaplan-Meier analysis of nude mice intracranially injected with $1 \times 10^{6} \mathrm{U} 87$ cells expressing $h \mathrm{HSS} 1(\mathrm{~N}=5)$, U87 wild-type $(\mathrm{N}=8)$ or mock-transfected cells $(\mathrm{N}=5)$ (Mantel test, $P<0.001$,). Survival and tumor growth were monitored daily

regulated by at least two-fold demonstrating that $h \mathrm{HSS} 1$ expression alters the gene expression profile of U87 cells. Table 1 displays 8 genes up or down-regulated by $h \mathrm{HSS} 1$.

$h$ HSS 1 expression in glioma-derived tissues

Next, we examined the expression of $h \mathrm{HSS} 1$ by transcript profiling in brain cancer and normal brain. The analysis of

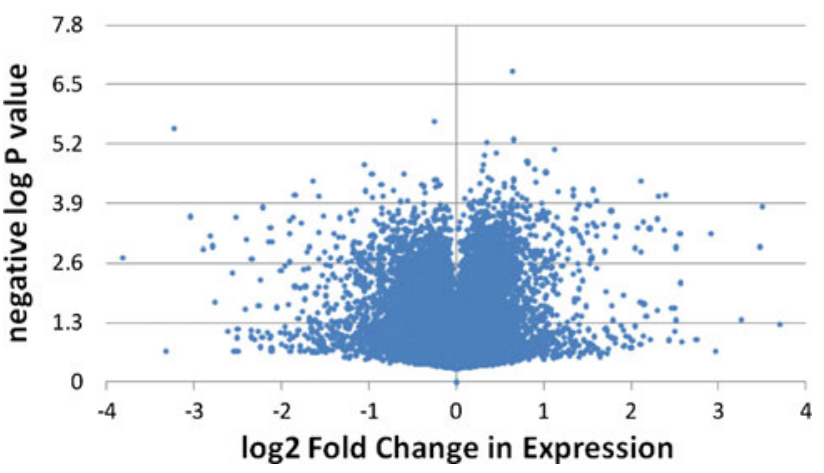

Fig. 8 Volcano plot for 491 differentially $h$ HSS1-regulated genes in U87 stable transfected cells. Values outside -1 and +1 fold change and values above negative $\log P$-value $1.3(P=0.05)$ were considered statistically significant down- (left) and up-regulated (right)

the Brain Cancer Tissue qPCR Array I showed expression in normal and all tumor grades with a decreased expression of $h \mathrm{HSS} 1$ in WHO grade IV (glioblastoma multiform) (Fig. 9a). Further, using the Human Brain Tissue qPCR Panel I $h$ HSS1 expression was found at low levels in normal tissues from various brain regions, with highest levels in pituitary tissue (Fig. 9b). Overall, the mRNA level of $h \mathrm{HSS} 1$ relative to the reference genes (GAPDH and $\beta$-actin) was very low in both surveys.

We also evaluated the distribution of $h \mathrm{HSS} 1$ in low-grade diffuse astrocytoma (WHO grade II) and highgrade astrocytoma (glioblastoma multiforme, WHO grade IV) using immunohistochemical analysis (Fig. 10). Overall, no obvious detection of $h \mathrm{HSS} 1$ was found in normal brain tissue (Fig. 10a and d). High expression levels of $h$ HSS1 were found in two out of four grade IV astrocytomas (Fig. 10c and f). On the contrary, all four grade II astrocytomas showed low expression of $h \mathrm{HSS} 1$ (Fig. 10b and e). A minimum perivascular staining was also observed, mostly in tumor areas. Although the expression of $h \mathrm{HSS} 1$ was predominantly detected in cytoplasm, nuclear stain was seen focally, as observed in A172 cells. Moreover, $h \mathrm{HSS} 1$ was detected in some but not all tumor cells. It is important to mention that both sets of primers used in the qRT-PCR and the antibody used to detect $h$ HSS1 do not discriminate between the secreted and membrane bound form of the protein ( $h \mathrm{HSM} 1)$.

$h \mathrm{HSS} 1$ expression in glioma molecular subclasses

Since $h$ HSS 1 expression was only detectable on a subset of high-grade gliomas, we considered whether $h$ HSS1 was differentially up-regulated on functionally distinct molecular subclasses of gliomas. Such subclasses are distinguishable by microarray, and include mesenchymal (MES), proneural (PN), and proliferative (Prolif) subclasses. In light of the $h \mathrm{HSS} 1$ transfection data, which suggested that 
Table 1 Select genes either up or down-regulated in U87 cells stably expressing $h$ HSS1 as indicated by microarray analysis

\begin{tabular}{|c|c|c|c|c|c|}
\hline \multirow[t]{2}{*}{ Gene } & \multirow{2}{*}{$\begin{array}{l}h \mathrm{HSS} 1 \text { vs pcDNA3.1 } \\
\text { Fold change }\end{array}$} & \multicolumn{3}{|l|}{$P$-value } & \multirow{2}{*}{$\begin{array}{l}\text { U87-wild type } \\
\text { vs pcDNA3.1 } \\
\text { Fold change }\end{array}$} \\
\hline & & $\begin{array}{l}h \mathrm{HSS} 1 \\
\text { vs U87-wild type }\end{array}$ & $\begin{array}{l}h \mathrm{HSS} 1 \\
\text { vs pcDNA3.1 }\end{array}$ & $\begin{array}{l}\text { U87-wild type } \\
\text { vs pcDNA3.1 }\end{array}$ & \\
\hline$h H S S 1 *$ & +11.7 & 0.00015 & 0.00013 & 0.39 & 1.0 \\
\hline$A P L N$ & -14 & 0.0004 & 0.00003 & 0.14 & 1.0 \\
\hline SIK1 & +2.3 & 0.003 & 0.00325 & 0.13 & -1.1 \\
\hline ADAMTS1 & +2.9 & 0.003 & 0.00203 & 0.23 & 1.0 \\
\hline BRCA1 & -2.7 & 0.0002 & 0.00032 & 0.12 & 1.0 \\
\hline$B R C A 2$ & -2.6 & 0.0007 & 0.00051 & 0.07 & -1.1 \\
\hline$C D K 2$ & -2.2 & 0.0008 & 0.00064 & 0.24 & 1.0 \\
\hline CDK6 & -3.1 & 0.001 & 0.00050 & 0.06 & -1.1 \\
\hline$A S F 1 B$ & -4.5 & 0.00005 & 0.00047 & 0.46 & 1.0 \\
\hline
\end{tabular}

$P$-values were determined by two-tailed paired Student's $t$-test. * Official gene symbol for $h \mathrm{HSS} 1$ is $C 19$ orf63

a

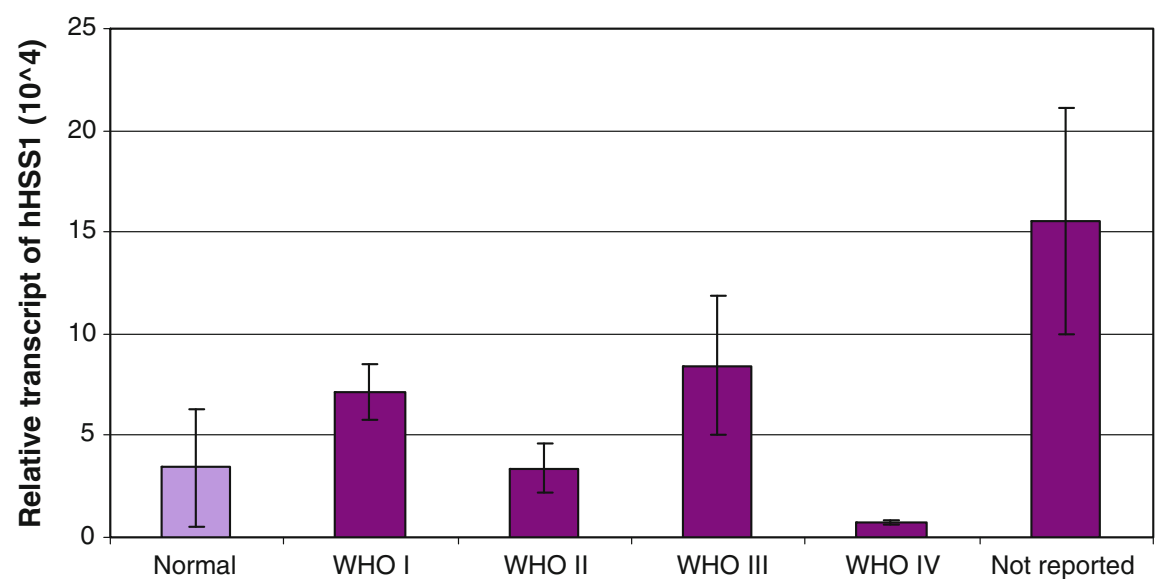

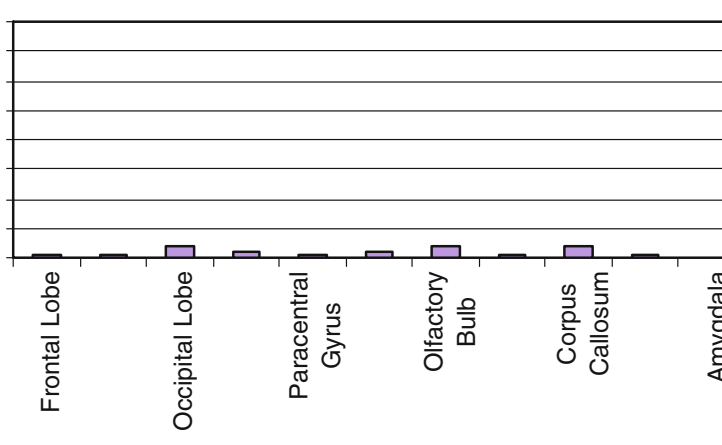

Fig. 9 a Expression profiles of $h \mathrm{HSS} 1$ in brain cancer according to WHO classification. TissueScan Brain Cancer Tissue qRT-PCR Array I consisting of 48 human brain tissues was used to determine transcript levels of $h \mathrm{HSS} 1$. Data were normalized to $\beta$-actin levels.

$h$ HSS1 expression may alter anchorage-dependent growth and/or cell morphology in vitro, it was particularly intriguing to consider whether $h \mathrm{HSS} 1$ was differentially expressed on MES subclass gliomas, which up-regulate genes involved in cell motility and invasiveness. Indeed,
Error bars displays the SEM. b Expression profiles of $h$ HSS 1 in human brain tissues (Human Brain Tissue qPCR Panel I). Data were normalized to GAPDH levels

relative up-regulation of $h \mathrm{HSS} 1$ expression uniquely characterized MES subclass gliomas, which expressed significantly more $h \mathrm{HSS} 1$ on microarray than either PN or Prolif subclass gliomas (Fig. 11). Since both MES and Prolif subclasses include de novo high-grade gliomas, 
Fig. 10 Immunohistochemical analysis of $h \mathrm{HSS} 1$ in representative cases of glioma. a, $\mathbf{d}$ No obvious immunoreactivity was detectable in normal brain tissues). b, e $h \mathrm{HSS} 1$ immunoreactivity was low in low-grade diffuse astrocytoma (WHO grade II). c, f low and high expression of $h \mathrm{HSS} 1$ in high-grade astrocytoma (glioblastoma multiforme, WHO grade IV). Sections were stained using anti-rabbit antibody against $h$ HSS 1 . Images are shown in the same magnification $(200 \times)$
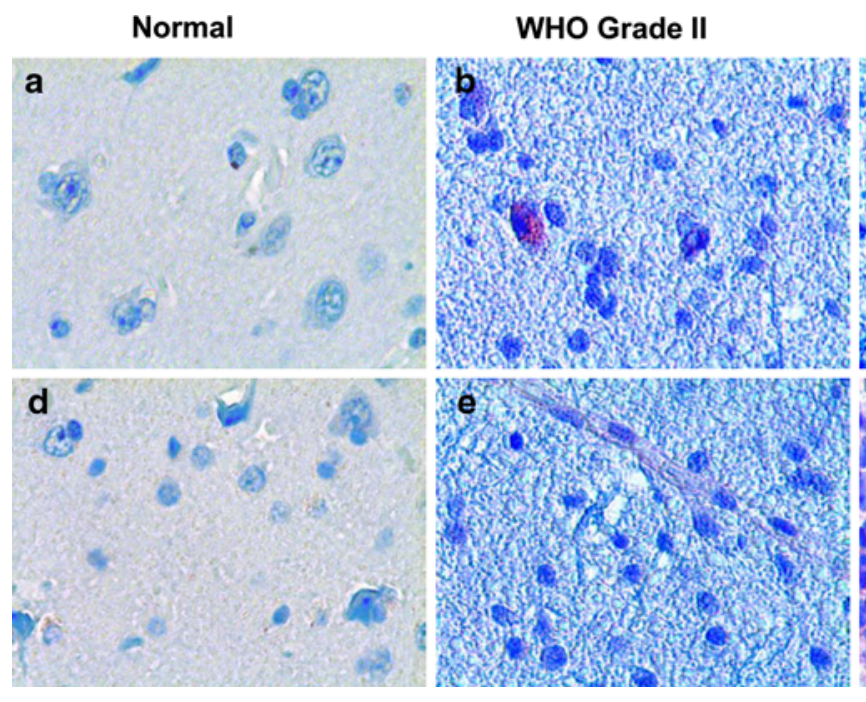

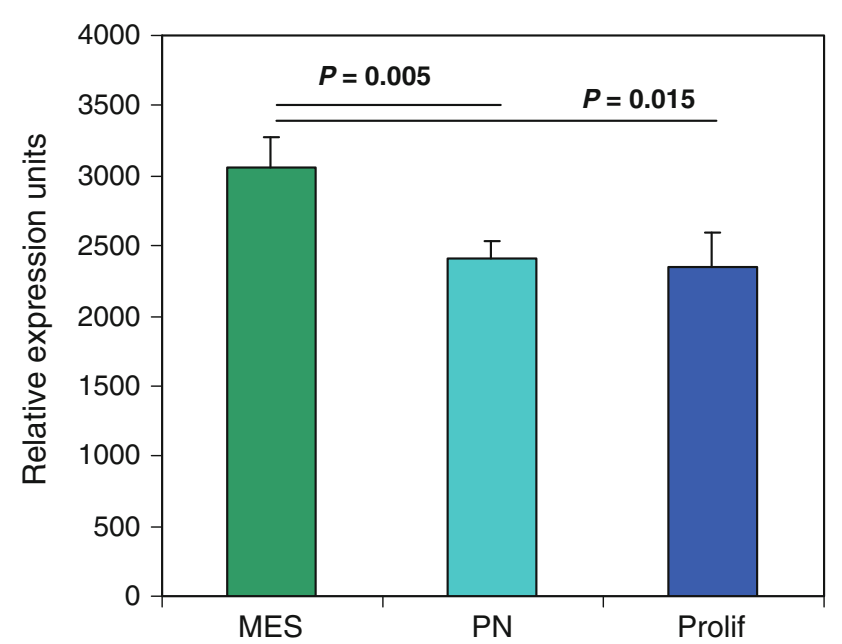

Fig. 11 Expression of $h \mathrm{HSS} 1$ by glioma molecular subclasses. Expression data from 100 primary gliomas from MD Anderson Hospital patients (GEO accession \#GSE4271) were arranged into Mesenchymal (MES; $\mathrm{n}=35$ ), Proneural (PN; $\mathrm{n}=37$ ), and Proliferative (Prolif; $\mathrm{n}=28$ ) groups according to Phillips et al. [8]. $h \mathrm{HSS} 1$ probeset (Affymetrix HG-U133, 224727_at) expression was assessed in each group from MAS5-normalized data. Statistical differences between groups was evaluated using one-tailed $t$-test after adjustment for variance, with $P$ values as shown. Mean expression \pm SEM was as follows: MES, $3057 \pm 211 ;$ PN, $2408 \pm 125$; Prolif, $2345 \pm 244$

whereas the PN subclass is largely devoid of these tumors, this finding further validates the notion that high $h \mathrm{HSS} 1$ expression is confined to a subset of high-grade gliomas generally, and to the MES subclass in particular.

\section{Discussion}

In this study, we have identified and characterized HSS1, a novel secreted factor, and its membrane-bound splice variant, HSM1. Moreover, we report that HSS1 and HSM1 are two members of a novel protein class, and that these novel proteins have growth inhibitory properties in two glioblastoma cell lines.

The fact that HSS1 and HSM1 are evolutionarily conserved across numerous species, phyla and even kingdoms, including mammals, invertebrates and plants, suggests that these proteins are of fundamental significance in biology. Yet neither human nor murine HSS1/HSM1 show homology to known, naturally occurring proteins, nor do they contain known functional domains. After using a 3-D crystallographic protein database to align protein sequences of unknown structure, it was found that a region common to HSS1/HSM1 shares structural homology with TOP-7, which is a non-natural, idealized protein designed in silico for stability at extreme temperature and $\mathrm{pH}$ conditions [9]. It is, therefore, intriguing to speculate whether HSS1/HSM1 might also exhibit such stability.

High-throughput gene expression analysis of diverse mouse and human tissue samples has shown that HSS1/ HSM1 is widely expressed in body tissues including brain [12]. We discovered HSS1/HSM1 by its differential expression pattern in highly purified HSCs, thus suggesting a possible role for this secreted factor in stem cell regulation. Interestingly, in a previous report $h \mathrm{HSS} 1 / \mathrm{HSM} 1$ was shown to be a component of the transcriptome involved in the signaling networks that control human embryonic stem cell growth and differentiation [15]. Numerous recent reports have revealed a connection between stem cell biology and tumor biology [16]; thus it is possible that HSS1 may have a role in both areas.

Gene expression data from the CGAP database does indicate that HSS1 may be involved in numerous cancers, such as brain, colon, eye, liver, lymph node, mammary gland, ovary, prostate and skin $[17,18]$. Moreover, Mitelman breakpoint data for the gene corresponding to HSS1 indicate that its gene locus is a "hot spot" for chromosomal 
aberrations implicated in a variety of human cancers [10], thereby suggesting a broad role for $h \mathrm{HSS} 1$ in cancer.

Upon the discovery of HSS1, only two facts guided the search for a function for this novel protein. First, HSS1 was highly expressed in HSCs and its human genetic locus was at $19 \mathrm{q} 13.33$. Since chromosome $19 \mathrm{q}$ also harbors at least one gene important for normal glial development and growth regulation as well as for the development of diffuse gliomas $[5,6,19]$, we chose to first investigate a possible role for $h$ HSS 1 in glioma. Another suggestive link between $h \mathrm{HSS} 1$ and glioma was that the common region for frequent deletions in glioma involves the 19q13.2-13.4 position [5]. This position is situated distal to the D19S178 marker and proximal to the D19S180 marker [5, 20], a region which includes the $h \mathrm{HSS} 1$ gene (Fig. 12).

Although chromosome 19q may harbor a potential panglioma tumor suppressor gene, to date, the glioma suppressor genes in this chromosome region remain elusive. Thus, we investigated the effect of ectopic expression of $h \mathrm{HSS} 1$ on the malignant properties of glioma-derived cells. The results showed that $h \mathrm{HSS} 1$ expression dramatically decreased the growth rate of U87 and A172 glioma-derived cells. In addition, stable expression of $h \mathrm{HSS} 1$ in A172 cells strongly inhibited their capacity to grow in low density compared with control cells, which might express endogenous $h \mathrm{HSS} 1$ at low levels. The ability to reduce their growth in low density was also observed for U87 cells stably transfected with $h \mathrm{HSS} 1$ (data not shown).

It was also striking that U87 cells expressing $h \mathrm{HSS} 1$ formed 5.9 times fewer cell aggregates compared to mocktransfected cells. The results also indicate that the expression of $h \mathrm{HSS} 1$ may promote growth suppression in U87 cells involving a decrease in growth rate following a decrease in the cell doubling-time. Further experiments are needed to clarify the mechanism by which $h \mathrm{HSS} 1$ suppresses the growth of U87 and A172 cells.

The expression of $h \mathrm{HSS} 1$ also significantly attenuated the colony-forming ability of U87 cells in soft agar matrix, further indicating a possible involvement of $h$ HSS 1 in restoration of contact inhibition. In addition, mice injected with U87 cells expressing $h$ HSS 1 showed on average greater than 2-fold extension of their life span, indicating that $h \mathrm{HSS} 1$ affected the malignant phenotype of gliomaderived cells.

Comparative expression profiling results using Affymetrix gene chip demonstrated that the stable expression of $h \mathrm{HSS} 1$ in U87 cells significantly altered its gene expression profile. A substantial number of genes were observed to be either up- or down-regulated by at least two-fold. Presumably genes among this population contribute to the phenotype observed for the $h$ HSS1 stable-transfected cells. Of immediate note and worth mention are the following observations. Apelin was seen as one of the most downregulated genes in $h \mathrm{HSS} 1$-overexpressing cells with an approximate 14-fold decrease in expression. Apelin expression has been observed to be highly up-regulated in the microvasculature in brain tumors. In particular, apelin has been shown to be needed for intersomitic vessel angiogenesis and the promotion of angiogenesis in brain tumors [21]. The fact that the expression of apelin is highly down-regulated in the $h \mathrm{HSS} 1$-overexpressing cells is consistent with the observed in vivo results where tumor growth was greatly suppressed, thereby leading to a significant increase in survival. It is of further interest that SIK1 (salt inducible kinase 1) gene was among the highly up-regulated genes. This finding is intriguing in that SIK1 has been shown to be a regulator of anoikis and a suppressor of metastasis [22]. Metastatic tumors generally will avoid anoikis despite loss of adherence. In the case of $h$ HSS1 stable-transfected cells, the increase in SIK1 would presumably correspond to an increase in anoikis and decrease in metastasis, thus suppressing tumor growth and spread. The ADAMTS1 gene was also up-regulated in $h$ HSS 1 stable-transfected cells. This metalloproteinase has been found to suppress tumorigenicity and metastasis in model systems [23, 24]. Further changes in gene expression

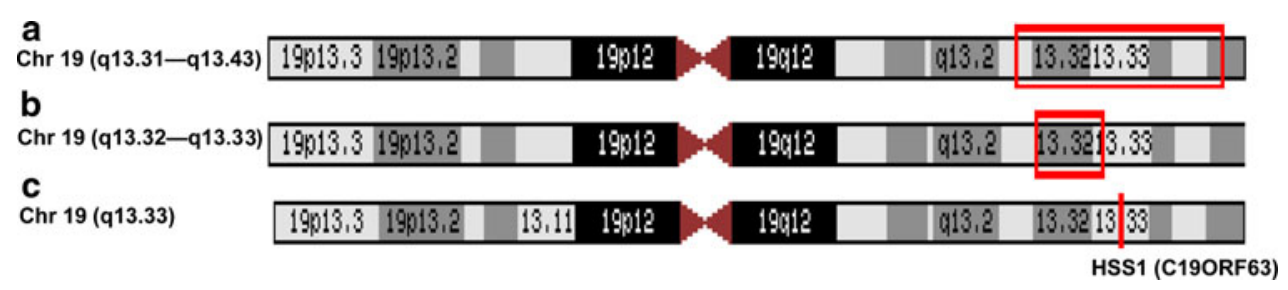

Fig. 12 Mapped position of the putative glioma tumor suppressor at chromosome 19q region a Chromosome 19 (q13.31-q13.43), region of the putative glioma tumor suppressor suggested by von Deimling et al. [20], between the markers D19S178 and D19S180 (red rectangle). This region includes the $h \mathrm{HSS} 1$ gene (C19orf63). b Chromosome 19 (q13.32-q13.33), region of the putative glioma tumor suppressor gene narrowed by Rubio et al. [5] (smaller red rectangle). This region is situated between the loci APOC 2 and HRC, which excludes the $h$ HSS1 gene. c Chromosome 19q13.33, red bar represent the genome location of $h \mathrm{HSS} 1$. The UCSC database was used as reference (http://genome.ucsc.edu/cgi-bin/hgGateway) 
to note in $h \mathrm{HSS} 1$-overexpressing cells include down-regulation of both BRCA1 and BRCA2 genes, a number of cell cycle associated genes (e.g. CDK2, CDK6), tubulins and the histone chaperone gene ASF1B along with a number of other histone genes. Validation of these and other genes of interest are underway.

In tumor tissues, the overall expression levels of $h \mathrm{HSS} 1$ were low and variable and the analysis of more tumor samples are needed to form a definite conclusion. However it is interesting to note that $h \mathrm{HSS} 1$ expression was observed minimally in all 4 low-grade diffuse astrocytomas (WHO grade II) analyzed, while half of the high-grade astrocytomas (glioblastoma multiforme, WHO grade IV) exhibited increased expression. This finding is in agreement with previous reports suggesting a correlation between low-grade gliomas and deletion of 19q13 region [25]; this deletion is less common in high-grade gliomas. Moreover, the notion that only a subset of high-grade gliomas over-expresses $h \mathrm{HSS} 1$ was further supported by microarray data, which indicated that MES subclass gliomas exclusively up-regulated $h \mathrm{HSS} 1$. This finding is intriguing in light of transfectant data that suggested stable expression of $h \mathrm{HSS} 1$ alters glioma anchorage-independent growth and morphology, thus implicating a functional role of $h \mathrm{HSS} 1$ in glioma growth and/or invasiveness. Further studies are required to test this hypothesis and to clarify if the high-expression of $h \mathrm{HSS} 1$ in high-grade gliomas are due to (a) mutations in $h \mathrm{HSS} 1$ itself; (b) mutations in downstream effectors of $h \mathrm{HSS} 1$; (c) interruption of pathways not related to $h \mathrm{HSS} 1$; and (d) detection of the splice variant of $h \mathrm{HSS} 1$ ( $h \mathrm{HSM} 1$ ).

Previous studies have suggested a few candidate glioma tumor suppressor genes in a 150-kilobase region that maps to $19 \mathrm{q} 13.33[5,6]$. It has been reported that alterations in GLTSCR1 are associated with the development and progression of oligodendroglioma [26]. Furthermore, p190RhoGAP, a gene located at 19q13.3, regulates critical components of PDGF oncogenesis and can act as a tumor suppressor in PDGF-induced gliomas by down regulation of Rho activity [27]. Our present studies suggest that $h \mathrm{HSS} 1$ is another factor located at $19 \mathrm{q} 13.33$ that is involved in suppression of glioma growth.

In conclusion, our data show that (i) $h \mathrm{HSS} 1$ is a novel, previously uncharacterized, secreted factor located within a previously defined candidate tumor suppressor gene region at $19 \mathrm{q} 13.33$ and (ii) $h \mathrm{HSS} 1$ is implicated in the growth inhibition and restoration of the normal contact inhibition properties of glioma-derived cells. Thus, the discovery of the novel secreted protein, $h \mathrm{HSS} 1$, and the elucidation of its functional role in the inhibition of glioma could provide the opportunity to develop novel treatment strategies for malignant gliomas.
Acknowledgments We thank to Joanne Wu and Audrey Izuhara for excellent technical support. This work was supported by Neumedicines Inc.

Open Access This article is distributed under the terms of the Creative Commons Attribution Noncommercial License which permits any noncommercial use, distribution, and reproduction in any medium, provided the original author(s) and source are credited.

\section{References}

1. Venter JC, Adams MD, Myers EW et al (2001) The sequence of the human genome. Science 291:1304-1351

2. Lander ES, Linton LM, Birren B et al (2001) Initial sequencing and analysis of the human genome. Nature 409:860-921

3. Pawlowski K (2008) Uncharacterized/hypothetical proteins in biomedical 'omics' experiments: is novelty being swept under the carpet? Brief Funct Genomic Proteomic 7(4):283-290. doi: 10.1093/bfgp/eln033

4. Zhong JF, Zhao Y, Sutton S et al (2005) Gene expression profile of murine long-term reconstituting vs. short-term reconstituting hematopoietic stem cells. PNAS 102:2448-2453

5. Rubio M-P, Correa KM, Ueki K et al (1994) The putative glioma tumor suppressor gene on chromosome $19 \mathrm{q}$ maps between $A P O C 2$ and $H R C^{1}$. Cancer Res 54:4760-4763

6. Smith JS, Tachibana I, Pohl U et al (2000) A transcript map of the chromosome 19q-arm glioma tumor suppressor region. Genomics 64(1):44-50

7. Zhao Y, Lin Y, Zhan Y et al (2000) Hematopoietic stem cell characterization and its regulation in BM transplantation. Blood 96:3016-3022

8. Phillips HS, Kharbanda S, Chen R et al (2006) Molecular subclasses of high-grade glioma predict prognosis, delineate a pattern of disease progression, and resemble stages in neurogenesis. Cancer Cell 9(3):157-173

9. Kuhlman B, Dantas G, Ireton GC, Varani G, Stoddard BL, Baker D (2003) Design of a novel globular protein fold with atomiclevel accuracy. Science 302:1364-1368

10. Mitelman F, Johansson B, Mertens F (eds) (2009) Mitelman database of chromosome aberrations in cancer. http://cgap.nci. nih.gov/Chromosomes/Mitelman

11. Law ME, Templeton KL, Kitange G et al (2005) Molecular cytogenetic analysis of chromosomes 1 and 19 in glioma cell lines. Cancer Genet Cytogenet 160:1-14

12. Su AI, Cooke MP, Ching KA et al (2002) Large-scale analysis of the human and mouse transcriptomes. Proc Natl Acad Sci USA 99:4465-4470

13. Vogler M, Giagkousiklidis S, Genze F, Gschwend JE, Debatin KM, Fulda S (2005) Inhibition of clonogenic tumor growth: a novel function of Smac contributing to its antitumor activity. Oncogene 24:7190-7202

14. Planque N (2006) Nuclear trafficking of secreted factors and cellsurface receptors: new pathways to regulate cell proliferation and differentiation, and involvement in cancers. Cell Commun Signal 4:7

15. Brandenberger R, Wei H, Zhang S et al (2004) Transcriptome characterization elucidates signaling networks that control human ES cell growth and differentiation. Nat Biotechnol 22:707-716

16. Ward RM, Dirks PB (2007) Cancer stem cells: at the headwaters of tumor development. Annu Rev Pathol 2:175-189

17. Lal A, Lash AE, Altschul SF et al (1999) A public database for gene expression in human cancers. Cancer Res 59:5403-5407 
18. Boon K, Osório E, Greenhut SF et al (2002) An anatomy of normal and malignant gene expression. Proc Natl Acad Sci USA 99:11287-11292

19. Smith I S, Tachibana HK, Lee J et al (2000) Mapping of the chromosome 19 q-arm glioma tumor suppressor gene using fluorescence in situ hybridization and novel microsatellite markers. Genes Chromosom Cancer 29:16-25

20. von Deimling A, Nagel J, Bender B et al (1994) Deletion mapping of chromosome 19 in human gliomas. Int $\mathbf{J}$ Cancer 57(5):676-680

21. Kälin RE, Kretz MP, Meyer AM et al (2007) Paracrine and autocrine mechanisms of apelin signaling govern embryonic and tumor angiogenesis. Dev Biol 305:599-614

22. Cheng H, Liu P, Wang ZC et al (2009) SIK1 couples LKB1 to p53-dependent anoikis and suppresses metastasis. Sci Signal 2:ra35

23. Kuno K, Bannai K, Hakozaki M et al (2004) The carboxyl-terminal half region of ADAMTS-1 suppresses both tumorigenicity and experimental tumor metastatic potential. Biochem Biophys Res Commun 319:1327-1333

24. Lee YJ, Koch M, Karl D et al (2010) Variable inhibition of thrombospondin 1 against liver and lung metastases through differential activation of metalloproteinase ADAMTS1. Cancer Res 70:948-956

25. Smith JS, Aderete B, Minn Y et al (1999) Localization of common deletion regions on $1 p$ and $19 q$ in human gliomas and their association with histological subtype. Oncogene 18:4144-4152

26. Yang P, Kollmeyer TM, Buckner K, Bamlet W, Ballman KV, Jenkins RB (2005) Polymorphisms in GLTSCR1 and ERCC2 are associated with the development of oligodendrogliomas. Cancer 103:2363-2372

27. Wolf RM, Draghi N, Liang X et al (2003) p190RhoGAP can act to inhibit PDGF-induced gliomas in mice: a putative tumor suppressor encoded on human chromosome 19q13.3. Genes Dev $17: 476-487$ 